\title{
Production of IL-12 from gene modified human dermal fibroblasts: a preclinical study for IL-12 cancer gene therapy
}

\author{
Chaehwa Park, ${ }^{1,5}$ Won-Ki Kang, ${ }^{1}$ Misook Oh, ${ }^{1}$ \\ Won Seog Kim, ${ }^{1}$ Jung-Hyun Yang, ${ }^{2}$ \\ Michael T. Lotze, ${ }^{3}$ Sunyoung Kim, ${ }^{4}$ \\ Keunchil Park ${ }^{1}$ and Chan H. Park ${ }^{1}$ \\ 1 Division of Hematology/Oncology, Samsung Medical Center, \\ Seoul 135-230, Korea \\ 2 Division of General Surgery, Samsung Medical Center, \\ Seoul 135-230, Korea \\ 3 University of Pittsburgh, School of Medicine, Pittsburgh, \\ PA 15261, U.S.A \\ 4 Institute for Molecular Biology and Genetics \\ Seoul National University, Seoul 151-742, Korea \\ 5 Corresponding author
}

Accepted 12 February 1997

Abbreviations: IL-12, interleukin-12; RT-PCR, reverse transcription-PCR

\begin{abstract}
Cytokine has been used as an immune stimulator and administered to patients for a treatment of cancer. Interleukin-12 (IL-12) is a potent cytokine which acts through a variety of functions including interferon- $\gamma$ production and cytotoxic T-cell activation. Considering the toxicity of high dose systemic IL-12 administration into human, local administration of low dose IL-12 can be a more efficient strategy. In ex vivo therapy, human dermal fibroblast has been considered as a useful vehicle for transferring genes. Here we show that human dermal fibroblast transduced with retrovirus containing IL-12 gene can be manipulated to produce reasonable amount of IL-12 protein. Human dermal fibroblast was isolated from freshly harvested skin specimens by collagenase digestion, grown in primary cultures, and transduced with a retroviral vector containing genes for human IL-12 and a selectable marker $\mathrm{Neo}^{\mathrm{R}}$. Following selection in G418, IL-12 producing fibroblasts were tested for secreted IL-12 level by ELISA. Six specimens of human skin were processed to obtain fibroblasts. ELISA results show that 40-150 units of IL-12 was produced for $24 \mathrm{~h}$ from $1 \times 10^{6}$ cells of transduced and selected fibroblast cultures. The primary cultures were maintained for up to nine passages about 108 days. The mean \pm overall time for obtaining enough
\end{abstract}

number of cells was $49 \pm 2$ days. The fibroblasts continued to produce IL-12 in culture for 90 days. These preliminary results can be used for the design of ex vivo gene therapy clinical trial using human dermal fibroblast.

Keywords: cancer gene therapy, human fibroblast, IL-12

\section{Introduction}

Recently, gene therapy appears to be a new way of treatment for cancer and many other genetic disorders. For most patients with disseminated and/or locally faradvanced cancer, conventional therapy can not be an efficient therapeutic modality. Murine therapeutic studies (Nastala et al., 1994; Tahara et al., 1994) demonstrated that interleukin-12 (IL-12) induces clinically significant tumor regression. This and many other reports imply that cytokine microenvironment at the tumor site may determine the outcome of the immune response (Tepper et al., 1989; Fearson et al., 1990; Gansbacher et al., 1990). Among those cytokines, IL-12 exerts a variety of biological effects on human $\mathrm{T}$ and NK cells which includes IFN- $\gamma$ production from peripheral blood lymphocytes and augmentation of allogeneic CTLresponses in combination with IL-2 (Kobayashi et al., 1989; Stern et al., 1990; Chan et al., 1991; Gately et al., 1991). The fact that IL-12 is produced only by professional antigen presenting cells like B cells or macrophages implies strongly that local administration of IL-12 at the tumor site may be an ideal strategy to elicit endogenous immune responses. With this method high concentration of IL-12 can be given locally without evolving systemic toxicity. Human skin fibroblast can be used as an efficient tool to transfer a gene ex vivo and finally to patient's tumor site for paracrine cytokine secretion. Human dermal fibroblast is a dividing cell, therefore, it is relatively easy to culture, transduce and select, and produce a relevant level of cytokine after gene transfer. The present study was designed to find out the level of individual variation in the IL-12 production after the culture, transduction, and selection of fibroblast as a preclinical study.

\section{Materials and Methods}

\section{Biopsies}

Six skin samples measuring $20 \mathrm{~cm}^{2}$ were obtained during surgical procedures performed on human adults. 


\section{Preparation of fibroblasts}

An ellipse of skin measure $10 \mathrm{~cm}$ by $2 \mathrm{~cm}$ is resected from a convenient location in a sterile fashion. The skin was transferred to laboratory and cleared of fat and epidermal layer. After dividing into 1-2 mm diameter pieces, the skin was incubated with $0.5 \%(\mathrm{w} / \mathrm{v})$ collagenase (Sigma Chemical, St. Louis, MO) and plated in flasks containing sterile culture medium and $10 \%$ human $A B$ serum. Culture medium contained fibroblast basal medium with $5 \mu \mathrm{g} / \mathrm{ml}$ human insulin (Life Technologies, Gaithersburg, MD), $1 \mathrm{ng} / \mathrm{ml}$ human bFGF (Clonetics, San Diego, CA), $50 \mu \mathrm{g} / \mathrm{ml}$ gentamycin, and $250 \mathrm{ng} / \mathrm{ml}$ amphotericin B. After first passage, fibroblasts were transduced with TFG-hIL-12-Neo retroviral vector. Protamine sulfate (Sigma Chemical) was added as carrier at a concentration of $5 \mu \mathrm{g} / \mathrm{ml}$. The stably transfected fibroblasts were grown in medium containing appropriate amount $(0.05-0.1 \mathrm{mg} / \mathrm{ml})$ of a neomycin analog, G418 (Geneticin, Life Technologies). All cultures were tested periodically to ensure the absence of mycoplasma contamination.

\section{Viral vector supernatants}

Retroviral vector containing genes of IL-12 and neomycine phosphotransferase (TFG-hIL-12-Neo retroviral vector) was obtained from M. Lotze of University of Pittsburgh (Zitvogel et al., 1994).

\section{Transduction and growth of fibroblasts}

Proliferating fibroblasts were transduced at a multiplicity of infection of 2 with the retroviral vector containing the genes for human IL-12 and neomycin phosphotransferase $\left(\mathrm{NeO}^{\mathrm{R}}\right)$. Transduction was performed for $2 \mathrm{~h}$ at $37^{\circ} \mathrm{C}$ in $5 \% \mathrm{CO}_{2}$ incubator. Forty-eight hours after transduction, they were exposed to the selective pressure of $0.05-0.2 \mathrm{mg} / \mathrm{ml}$ of G418.

\section{IL-12 detection}

IL-12 in culture supernatants was measured using an ELISA kit purchased from R \& D Systems (St. Louis, $\mathrm{MO})$. Culture medium of fibroblasts was collected periodically as described in Figure 3 . IL-12 levels were expressed as $\mathrm{ng} / 24 \mathrm{~h}$ per $1 \times 10^{6}$ fibroblasts.
Insertion of TFG-hIL-12-Neo retroviral DNA into fibroblasts was confirmed by PCR. PCR condition was the same as suggested by manufacturer (Perkin Elmer, Branchburg, NJ). The sets of $5^{\prime}$ and $3^{\prime}$ primers used were 5'-GGTATCACCTGGACCTTGG-3' and 5'-GCTGCAAGTTGTTGGGTGG-3' for p40 subunit of IL-12, 5'-ATGATTGAACAAGATGGATTGCAC-3' and 5'-TTCGTCCAGATCATCCTGATCGAC-3' for neomycin phosphotransferase (neo) and 5'-TTGTCCACCACGGTGCTCAAT-3' and 5'-GGCTCGTACTCTATAGGCTTC-3' for envelope gene (env). The expected length of PCR products are $540 \mathrm{bp}, 450 \mathrm{bp}$ and 712 bp, respectively.

\section{Reverse transcription (RT)-PCR for IL-12}

Expression of IL-12 mRNA in transduced fibroblasts was confirmed using RT-PCR. mRNA was extracted from approximately $1 \times 10^{7}$ transduced cells using a single step RNA extraction methods with TRIzol (Gibco BRL, Gaithersburg, MD). RT-PCR codition was the same as suggested by manufacturer (Perkin Elmer). The sets of 5'and 3' primers for PCR were 5'-GGTATCACCTGGACCTTGGACCAGAGC-3' and 5'-GCTGCAAGTTGTTGGGTGGGTCAGGTTTG-3' for p40 subunit of IL-12 and 5'-ATGATTGAACAAGATGGATTGCAC-3' and 5'-TTCGTCCAGATCATCCTGATCGAC-3' for neo. The expected length of PCR product is 540 and $450 \mathrm{bp}$, respectively.

\section{Results}

\section{Culture of fibroblasts}

Primary fibroblasts has been reported to show higher growth rate in suspension culture comparing to the conventional explant culture (Veelken et al., 1994). As shown in Table 1, there were differences in growth rate of individual specimen. Specimen-1 and 2 were growing very well, while specimen-4 stopped growing around day 70 after delayed growth and specimen-5 showed considerably low growth rate comparing to other ones. As demonstrated by Veelken et al. (1994), most of the cultures eventually underwent a retardation of proliferation. And the overall cell number that could be reached until retardation of growth correlated with the age of donor (Table 1).

\section{Transduction of a TFG-hIL-12-Neo retrovirus}

PCR for IL-12, neo and env

Table 1. Amount of produced IL-12 after transduction and selection of human dermal fibroblast in culture.

\begin{tabular}{lcccccc}
\hline Specimen No. & 1 & 2 & 3 & 4 & 5 & 6 \\
\hline Sex(age) of donor & $\mathrm{M}(49)$ & $\mathrm{F}(32)$ & $\mathrm{F}(64)$ & $\mathrm{M}(54)$ & $\mathrm{M}(58)$ & $\mathrm{M}(64)$ \\
Fibroblasts & 7.6 & 11.7 & 4.4 & 1 & 1.3 & 1.8 \\
at 1st passage $\left(\times 10^{6}\right)$ & $150 \pm 10$ & $40 \pm 12$ & $100 \pm 7$ & $66 \pm 2$ & $40 \pm 5$ & $67 \pm 5$ \\
\hline $\mathrm{IL}-12\left(\mathrm{ng} / 10^{6} / 24 \mathrm{~h}\right)$ & 150 \\
\hline
\end{tabular}




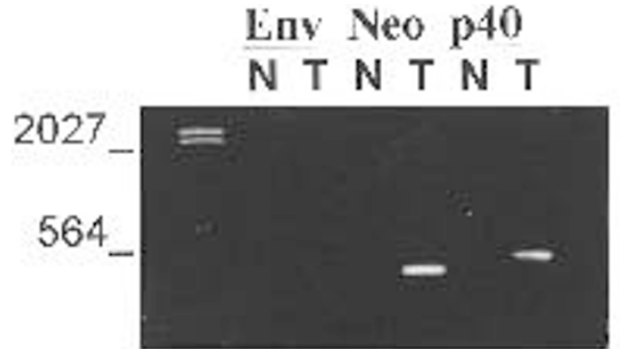

Figure 1. Gene transfer of IL-12 containing retroviral vector into cultured human fibroblast. PCR analysis was performed with DNA from transduced $(T)$ and nontransduced (N) fibroblast. Env, envelope of amphotropic retrovirus; Neo, neomycin phosphotransferase; p40, p40 subunit of IL-12.

\section{into human fibroblast}

To assess the transfer of the human IL-12 gene into the fibroblast, PCR was performed using genomic DNAs from fibroblasts before and after transduction. Successfully transduced fibroblasts gave amplified products of $540 \mathrm{bp}$ with primers for IL-12 and $490 \mathrm{bp}$ with primers for neo gene, as shown in Figure 1. Transduced fibroblasts didn't show positive band of 712 bp with retrovirus env gene primers after transduction.

\section{IL-12 mRNA and protein are expressed in transduced cells}

To generate fibroblasts secreting IL-12, retroviral vectors expressing p35 and p40 subunit of IL-12 and neomycin phosphotransferase (crip-TFG-hIL-12-Neo) were given to transduce human fibroblasts. Following transduction, selection in G418, and expansion of G418 resistant cells, the supernatant was tested for IL-12 secretion by ELISA. RT-PCR of transduced cells showed expression of both IL-12 p40 subunit and neomycine phosphotransferase in transfected fibroblast but expression of neither mRNA was detected in untransfected one (Figure 2).

\section{Interleukin-12 production profiles of transduced fibroblasts}

After selection with G418, non-transduced cells were dying while transduced fibroblasts were selectively growing very well (Figure 3). IL-12 production profile of specimen-2 fibroblasts was shown in Figure 4. All other specimens had similar profiles of $\mathrm{IL}-12$ production and none of six normal fibroblasts secreted detectable IL-12 production. As shown in the figure, the fibroblasts number continued to increase after culture until G418 selection began around day 15 , day 32 and day 47 . Then, during G418 selection, the growth rate of fibroblasts was reduced dramatically until the end of each selection step (day 25, day 37 and day 52 ). After the selection was completed around day 52 , fibroblasts number began to increase again. The selection of transduced

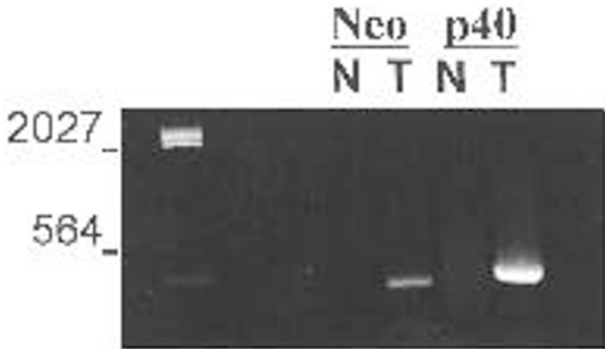

Figure 2. Expression of IL-12 mRNA in fibroblasts transduced with crip-TFG-hlL-12Neo retrovirus. Reverse transcription-PCR was performed for mRNA harvested from transduced and untransduced human fibroblasts. N, mRNA from human fibroblasts; T, mRNA from transduced and selected human fibroblasts.

A

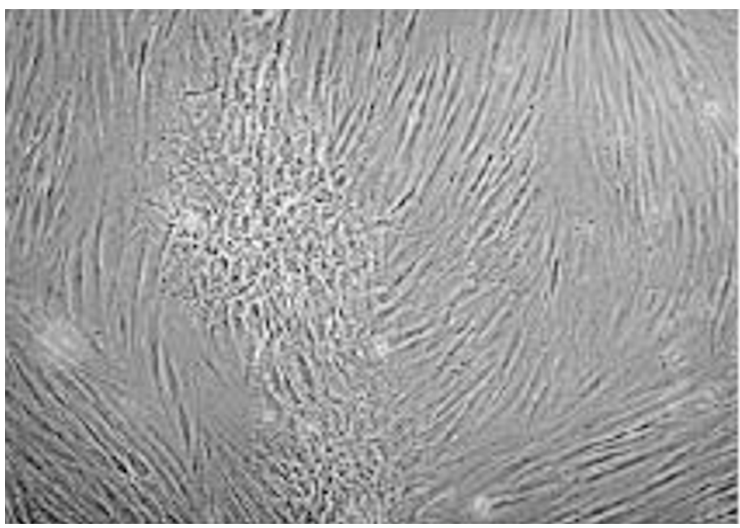

B

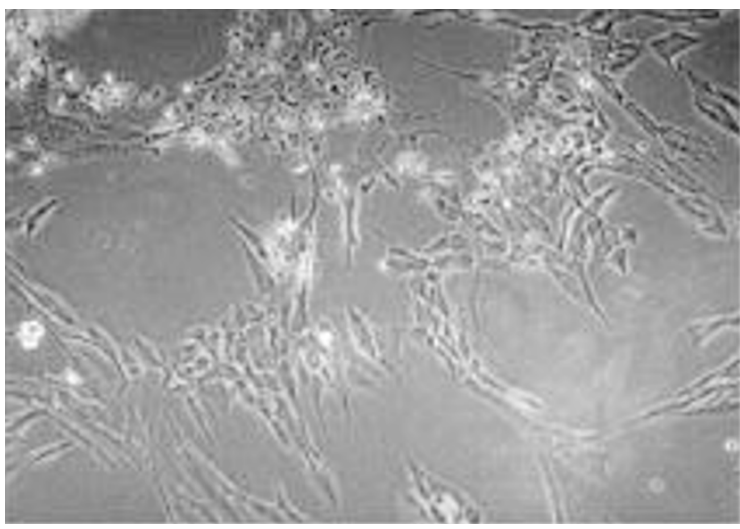

Figure 3. Fibroblast before and after selection with G418. A, Before selection. B, After selection.

fibroblasts coincides with the increase in IL-12 production as shown in Figure 4. Although there was some differences among individual specimens, IL-12 was produced in a reasonable amount $(40-150 \mathrm{ng} / 24$ $\mathrm{h} / 10^{6}$ cells) in all specimens. 


\section{Discussion}

Retroviral vector used here has three cistrons, p35, p40 subunits for IL-12 and neo as a selection marker (Zitvogel et al., 1994). Figure 1 shows that all these genes are integrated stably to the fibroblasts. Duration of cytokine secretion in vitro was determined after transfection. IL-12 secretion of retrovirally transfected fibroblasts initially increased upto about $70 \mathrm{ng}$ per $1 \times$ $10^{6}$ cells for $24 \mathrm{~h}$ (Figure 4) and was measurable for 12 weeks (data not shown). There were some differences in the amount of secreted IL-12 according to individual as shown in Table 1. The range of IL-12 was between 40 to $150 \mathrm{ng}$ per $1 \times 10^{6}$ cells for $24 \mathrm{~h}$. These amounts are enough for phase I clinical trial which has IL-12 of 10- 3,000 ng range for $24 \mathrm{~h}$.

IL-12 induces a considerably increased production of IFN- $\gamma$ from peripheral blood lymphocytes after slight increase in IL-12 production. Nastala et al. (1994) reported that some animals cured by IL-12 did not have long-term immunity and the anti-tumor effect of IL-12 may include non-T cell-mediated immune reactions. Data published by Voest et al. (1985) can be one of these examples. They suggested that anti-angiogenic effects of IL-12 may account for antitumor activity.

In several clinical situations, transient expression of a therapeutic molecule by genetically modified cells might offer advantages over the application of the recombinant gene product. First, systemic toxicities associated with immunotherapy might be overcome if cytokine expression is localized to the tumor site. Bramson et al. (1996) have reported that IL-12 expression is localized to tumor after injection. They also showed that the virally expressed cytokine is produced at high levels within the tumor and remains localized to the site, indicating that systemic complications due to excess IL-12 in the serum can be overcome. Second, local delivery of cytokines is closer to the natural immune response. Zitvogel et al. (1994) demonstrated that fibroblasts transduced with a retroviral vector expressing IL-12 can be used to treat pre-established tumors in mouse model. They reported that antitumor effects were observed more rapidly when the cells were injected at the tumor site.

IL-12 has been proven as an useful therapeutic agent for cancer gene therapy in a mouse model using retroviral vector (Tahara et al., 1994). Bramson et al. (1996) have demonstrated that in mouse model using adenoviral system expressing IL-12, the serum IL-12 decline five-fold from day 1 to day 3 whereas the intratumoral IL-12 only began to decline around day 6 . All these results indicate that local expression is more effective.

In human, even after lethal irradiation, tumor itself can not be an adequate source for the production of therapeutic molecules. Among many known potential target cell types for gene delivery, like endothelial cells, myoblasts, and hepatocytes (Wilson et al., 1989; Barr et al., 1991; Dhawan et al., 1991), fibroblasts are chosen because it is easy to culture, transfect with retrovirus and select with G418 (Garver et al., 1987; Palmer et al., 1987; Selden et al., 1987; Moullier et al., 1993; Heartlein et al., 1994; Naffakh et al., 1995). IL-12 secretion can be maintained in vitro for more than 90 days (data not shown), indicating that stably transfected fibroblasts that secrete sufficient amount of transfected gene product can be useful for cancer gene therapy.

\section{Acknowledgments}

We thank Dr. Hideaki Tahara for the gift of the TFGhIL-12-Neo vector.

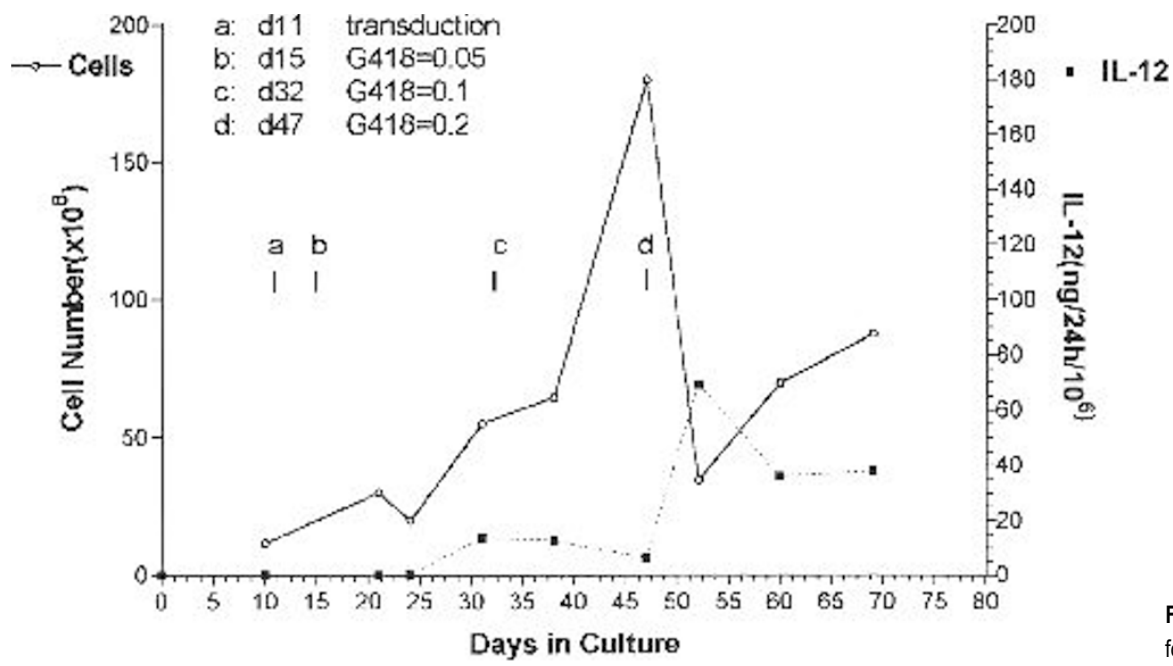

Figure 4. Profile of IL-12 production specimen-2 following selection with G418. 


\section{References}

Barr, E. and Leiden, J. M. (1991) Systemic delivery of recombinant proteins by genetically modified myoblasts. Science 254: 1507-1509

Bramson, J. L., Hitt, M., Addison, C. L., Muller, W. J., Gauldie, J. and Graham, F. L. (1996) Direct intratumoral injection of an adenovirus expressing interleukin-12 induces regression and long-lasting immunity that is associated with highly localized expression of interleukin-12. Hum. Gene Ther. 7: 1995-2002

Chan, S. H., Perussia, B., Gupta, J. W., Kobayashi, M., Pospisil, M.,Young, H. A.,Wolf, S. F., Young, D., Clark, S. C. and Trinchieri, G. (1991) Induction of Interferon- $\gamma$ production by natural killer cell stimulatory factor: characterization of the responder cells and synergy with other inducers. J. Exp. Med. 173: 869-879

Dhawan, J., Pan, L. C., Pavlath, G. K., Travis, M. A., Lanctot, A. M. and Blau, H. M. (1991) Systemic delivery of human growth hormone by injection of genetically engineered myoblasts. Science 254: 1509-1512

Fearon, E. R., Pardoll, D. M., Itaya T., Golumbek., Levitsky, H. I., Simons, J. W., Karasuyama, H., Vogelstein, B. and Frost, P. (1990) Interleukin-2 production by tumor cells bypass $T$ helper function in the generation of an antitumor response. Cell 60: 397403

Gansbacher, B., Zeier, K., Daniels, B., Cronin, K., Bennerji, R. and Gilboa, E. (1990) Interleukin 2 gene transfer into tumor cells abrogates tumorigenicity and induces protective immunity. J. Exp. Med. 172: 1217-1224

Garver, R. I, Chytil, A., Courtney, M. and Crystal, R. (1987) Clonal gene therapy: Transplanted mouse fibroblast clones express human $\alpha_{1}$-antitrypsin gene in vivo. Science 237: 762-764

Gately, M. K., Desai, B. B., Wolitzky, A. G., Quinn, P. M., Dwyer, C. M., Podlaski, F. J., Familletti, P. C., Sinigalia, F. Chizzonite, R., Gubler, U. and Stern, A. S. (1991) Regulation of human lymphocyte proliferation by a heterodimeric cytokine, IL-12 (cytotoxic lymphocyte maturation factor). J. Immunol. 147: 874-882

Heartlein, M. W., Treco, D. A. and Selden, R. (1994) Long-term production and delivery of human growth hormone in vivo. Proc. Natl. Acad. Sci. USA 91: 10967-10971

Kobayashi, M., Fitz, L., Ryan, M., Hewick, R. M., Clark, S. C., Chang, S., Loudon, R., Sherman, F., Perussia, B. and Trinchieri, G. (1989) Identification and purification of natural killer cell stimulatory factor (NKSF), a cytokine with multiple biologic effects on human lymphocytes. J. Exp. Med. 170: 827-845

Naffakh, N., Henri, A., Villeval, J. L., Rouyer-Fessard, P., Moullier, P., Blumenfeld, N., Danos, O., Vainchenker, W., Herad, J. M. and Beuzard, Y. (1995) Sustained delivery of erythropoietin in mice by genetically modified skin fibroblasts. Proc. Natl. Acad. Sci. USA 92: 3194-3198

Nastala, C. L., Edington, H. D., Mckinney, T. G., Tahara, H., Nalesnik, M. A., Brunda, M. J., Gately, M. K., Wolf, S. F., Schreiber, R., Storkus, W. J. and Lotze, M. T. (1994)
Recombinant interleukin-12 administration induces tumor regression in association with IFN- $\gamma$ production. J. Immunol. 153: 1697-1706

Moullier, P., Bohl, D., Heard, J. M. and Danos, O. (1993) Correction of lysosomal storage in the liver and spleen of MPS VII mice by implantation of genetically modified skin fibroblasts. Nature Genet. 4: 154-159

Palmer, T. D., Hock, R. A., Osborne, W. R. A. and Miller, A. D. (1987) Efficient retrovirus-mediated transfer and expression of human adenosine deaminase gene in diploid skin fibroblasts from an adenosine deaminase-deficient human. Proc. Natl. Acad. Sci. USA 84: 1055-1059

Selden, R. F., Skosjiewicz, M. J., Howie, K. B., Russel, P. S. and Goodman, H. W. (1987) Implantation of genetically engineered fibroblasts in mice. Science 236: 714-718

Stern, A. S., Podlaski, F. J., Hulmes, J. D., Pan, Y. C. E., Quinn, P. M, Wolitzky, A. G. Familletti, P. C., Stremlo, D. L., Truitt, T., Chizzonite, R. and Gately, M. K. (1990) Purification to homogeneity and partial characterization of cytotoxic lymphocyte maturation factor from human B-lymphoblastoid cells. Proc. Natl. Acad. Sci. USA 87 6808-6812

Tahara, H., Zeh, H. J. Z. III, Storkus, W. J., Pappo, I., Watkins, S. C., Gubler, U., Wolf, S. F., Robbins, P. D. and Lotze, T. M. (1994) Fibroblasts genetically engineered to secrete interleukin-12 can suppress tumor growth and induce anti-tumor immunity to a murine melanoma in vivo. Cancer Res. 54: 182-189

Tepper, R. I., Pattengal, P. K. and Leder, P. (1989) Murine interleukin-4 displays potent anti-tumor activity in vivo. Cell 57: 503-512

Veelken, H., Jesuiter, H., Mackensen A., Kulmburg P., Schultze, J., Felicia, R. Mertelsmann, R. and Lindemann, A. (1994) Primary fibroblasts from human adults as target cells for ex vivo transfection and gene therapy. Hum. Gene Ther. 5: 1203-1210

Voest, E. E., Kenyan, B. M., O'Reilly, M. S., Truitt, G., D’Amato, R. J. and Folkman, J. (1995) Inhibition of angiogenesis in vivo by interleukin 12. J. Natt. Cancer Inst. 87: 581 586

Zitvogel, L., Tahara, H., Ca, Q., Storkus, W., Muller, G., Wolf, S. F., Gately, M. K. Robbins, P. D. and Lotze, M. T. (1994) Construction and characterization of retrovira vectors expressing biologically active human Interleukin-12. Hum. Gene Ther. 5: 14931506 